\title{
Aplicación del análisis de imágenes al estudio del pre-tratamiento y temperatura en el pardeamiento enzimático rodajas de manzana
}

\section{Application of the analysis of images to the study of the pre-treatment and temperature in the enzymatic browning of apple slices}

\author{
Lorenzo Cabañas Chávez ${ }^{1}$, Wilson Manuel Castro Silupu ${ }^{2}$
}

\section{RESUMEN}

El presente trabajo tuvo como objetivo evaluar el efecto de la temperatura y el pre-tratamiento en el color en rodajas de manzana var. Granny Smith, medidos en el espacio CIELa*b* utilizando un sistema de visión por computadora, durante el proceso de pardeamiento enzimático. La metodología aplicada consto' de las siguientes actividades: a) Aplicación de diferentes condiciones de temperatura $\left(10,25\right.$ y $40^{\circ} \mathrm{C}$ ) y pre- tratamiento (control, escaldado y sulfitado) a rodajas de manzana para analizar el pardeamiento enzimático, b) Extracción de información del color durante el proceso de pardeamiento enzimático y c) Relacionar los cambios en el color, la temperatura y el pre-tratamiento. Los resultados de la labor experimental muestran que a lo largo del proceso de pardeamiento se produce la reducción de los parámetros L e incremento de parámetros a* y b*; asimismo, que existen diferencias significativas en los parámetros $\mathrm{L}, \mathrm{a}^{*} \mathrm{y} \mathrm{b}^{*}$ cuando se aplican diferentes pre-tratamientos y temperaturas. Finalmente, las ecuaciones, que modelan las relaciones entre los parámetros L, a* y b*, en función de los factores pre-tratamiento (P), tiempo (Tp), y temperatura de almacenamiento (Ta) confirman que los parámetros $\mathrm{L}, \mathrm{a}^{*} \mathrm{y} \mathrm{b}$ * tienen relación con los factores en evaluación. Sin embargo, por los bajos valores de R2, no es posible utilizarlos para predecir el proceso.

Palabras clave: Análisis de imágenes, CIELa*b*, pardeamiento, visión por computadora

\begin{abstract}
The present work had as objective evaluate the effect of temperature and pre- treatment in color apple slices var. Granny Smith, measured it in CIELa*b* space using a computer vision system, during the process of enzymatic browning. The applied methodology consists of the following activities a) Application of different conditions of temperature $\left(10,25\right.$ and $40^{\circ} \mathrm{C}$ ) and pre-treatment (control, blanching and sulphited) to apple slices to analyze enzymatic browning, b) Extraction of information color during the process of enzymatic browning and c) relate the changes in color, temperature and pre-treatment. The results show that the experimental work along the browning process occurs reduction and increased parameters $\mathrm{L}, \mathrm{a}^{*}$ and $\mathrm{b}^{*}$ parameters, also significant differences in the parameters $\mathrm{L}, \mathrm{a}^{*}$ and $\mathrm{b}^{*}$ when applied different pre-treatments and temperatures. Finally, the equations that model the relationship between the parameters $\mathrm{L}, \mathrm{a}^{*}$ and $\mathrm{b}^{*}$, based on the factors pre-treatment (P), time (Tp), and storage temperature (Ta) confirm that the parameters $\mathrm{L}, \mathrm{a}^{*} \mathrm{~b}^{*}$ are related to the evaluation factors. However, the lower values of 2 , it is not possible to use them to predict the process.
\end{abstract}

Keywords: Image analysis, CIELa*b*, browning, computer vision

${ }^{1}$ Bachiller en Ingeniería Agroindustrial email: lorecab_10@aoutlook.com

${ }^{2}$ Docente Asociado, Facultad de Ingeniería Zootecnista, Agronegocios y Biotecnología, UNTRM email:wilson.castro@untrm.edu.pe 


\section{INTRODUCCIÓN}

El aspecto y el color de la superficie del alimento es el primer parámetro de calidad evaluado por los consumidores y es crítico en la aceptación del producto (Xul y col., 2009). Por tanto; es frecuentemente necesario el analizar el color superficial de muestras alimentarias y expresar este cualitativa o cuantitativamente, mediante distribución del color y su representación en forma de histogramas (Kit y Spyridon 2004).

Sin embargo; la mayoría de los instrumentos comerciales usados en la determinación del color no son adecuados para la investigación en Ingeniería de Alimentos, ya que están diseñados principalmente para el control de calidad y sólo proporcionan valores medios (Briones y Aguilera 2005; Kit y Spyridon 2004).

Ante estas dificultades se han desarrollado otros métodos de análisis, tales como los basados en sistemas de visión por computador para el análisis de color de los alimentos Kang y Sabarez (2009), Briones y Aguilera (2005) y Du y Sun (2005). Estos sistemas, como lo muestran Lunadei y col. (2011), León (2005), han sido usados para medir objetivamente el color de diferentes alimentos debido a que permiten la adquisición de imágenes de muestras completas, que pueden ser analizadas pixel por pixel, permitiendo una medida más aproximada en cada punto de la superficie y la cuantificación de las características superficiales y defectos. Una limitante a estos sistemas es el empleo de equipo especializado o algoritmos, no accesibles a la mayoría de los investigadores, y la dependencia de las condiciones externas a las cuales se toman las imágenes, tales como la iluminación. (Du y Sun, 2005; Kit y Spyridon 2004).

Con todo lo anterior existen interesantes trabajos relacionados con las mediciones de las propiedades de materiales alimentarios y el análisis de imágenes (Lunadei y col.2011; Kang y Sabarez 2009; Du y Sun, 2005; Briones y Aguilera 2005; Paschos y Petrou 2003) tanto para medir las características de color u morfológicas como para relacionar estas con las propiedades del material.

Una reciente opción al análisis en el espacio CIEL*a*b* es el uso de histogramas para analizar imágenes. Esto debido a que tiene las ventajas de no ser sensitivos a la rotación, escalado y pequeñas deformaciones de los objetos e inmune al ruido. Otro mérito de los histogramas, el cual atrae nuestro interés para el desarrollo de sistemas de análisis en tiempo real y sus operaciones es su gran simplicidad reduciendo significativamente los costos computacionales (Brosnan y Sun, 2004). Las buenas propiedades mencionadas arriba han llevado a muchos investigadores al desarrollo de algoritmos basados en los histogramas. Estos histogramas pueden ser utilizados directamente caracterizando a este mediante algunas de sus propiedades, tales como los picos principales, momentos, etc., o comparación de los histogramas de diferentes imágenes con fines de reconocimiento (Guoping y col. 2004; Paschos y Petrou 2003; Yoo y Il-Seok 1999).

Por tanto; el objetivo del presente trabajo fue evaluar el análisis de imágenes, con base en el espacio de color CIELa*b* y la aplicación de histogramas, al estudio del pre-tratamiento y la temperatura en el pardeamiento enzimático de rodajas de manzana.

\section{MATERIAL Y MÉTODO}

\subsection{Materia prima}

Muestras de manzanas Var. Granny Smith fueron adquiridas en el mercado local de la ciudad de Chachapoyas y determinando visualmente que estas muestras se encuentren en estado de madurez comercial, véase Figura 1.

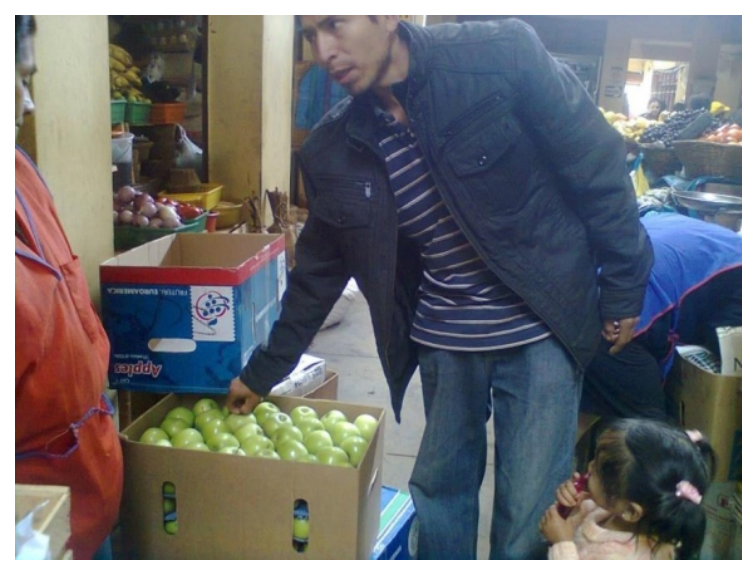

Figura 1.Adquisición de muestras de manzanas

\subsection{Reactivos}

Los reactivos usados fueron agua oxigenada en solución al 5 \% y Bisulfito de sodio (NaHSO3), grado reactivo.

\subsection{Equipos}

Los equipos usados fueron congeladora con rango de temperatura de -20 a $5^{\circ} \mathrm{C}$, equipo de sistema de visión computacional (Figura 2) y una estufa de secado con rango entre 10 y $120^{\circ} \mathrm{C}$. 


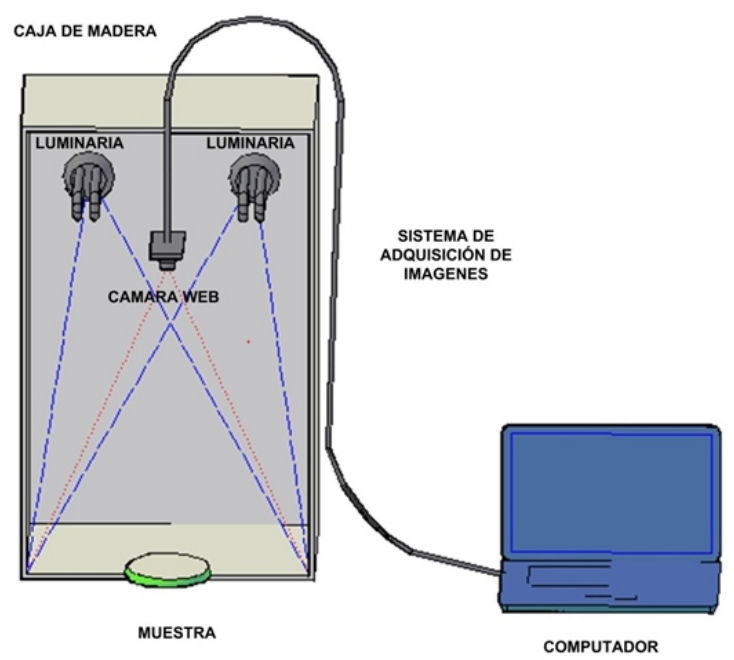

Figura 2. Sistema de visión por computadora usado en la investigación

\section{TRABAJO EXPERIMENTAL}

Los métodos utilizados en esta investigación se describen a continuación:

Adquisición de imágenes de rodajas de manzana sometidas a diferentes condiciones de temperatura y pre-tratamiento. Las muestras fueron adquiridas en el mercado local, realizando inspección visual del estado de calidad. Posteriormente fueron lavadas y se cortaron discos de $0.5 \mathrm{~cm}$ de espesor, en sentido transversal al eje y a media altura del mismo, Figura 3.

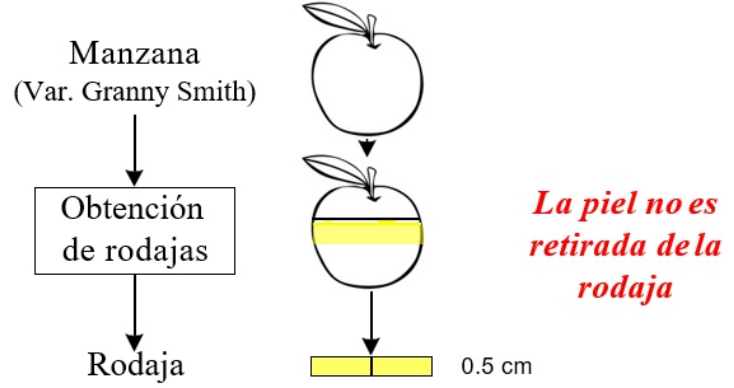

Figura 3. Esquema de obtención de rodajas de manzanas

Las rodajas fueron pre tratadas según como se describe en los siguientes puntos: a) muestras sin tratamiento b) muestras escaldadas, a $70{ }^{\circ} \mathrm{C}$, por 1 minuto y c) muestra sulfitada, inmersión por 1 minuto en solución de bisulfito de sodio $0.05 \mathrm{~g} / 100$ $\mathrm{ml}$. Finalmente las muestras fueron ubicadas en el sistema de adquisición de imágenes, mostrado en la Figura 2, registrando imágenes cada 30 minutos hasta alcanzar 4 horas.
Obtención de parámetros de color durante el proceso de pardeamiento enzimático. En la determinación de parámetros de color se desarrolló un programa en el software matemático Matlab 2015 a el cual separa las labores de determinación de los parámetros de color en los siguientes pasos:

Preprocesamiento y segmentación. Las imágenes en formato .JPG, son segmentadas, Figura 4a y Figura $4 \mathrm{~b}$, aislando los elementos de interés, imagen de la manzana, del fondo determinando que pixeles contienen la información de color y morfología.

Determinación de parámetros de color. Esta función analiza el color de la imagen segmentada. Posteriormente; los valores numéricos de cada pixel del elemento de interés, así como su histograma son obtenidos, Figura 4c.

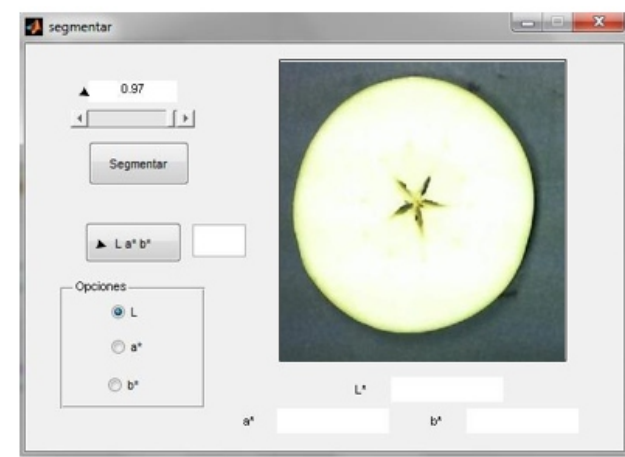

a)

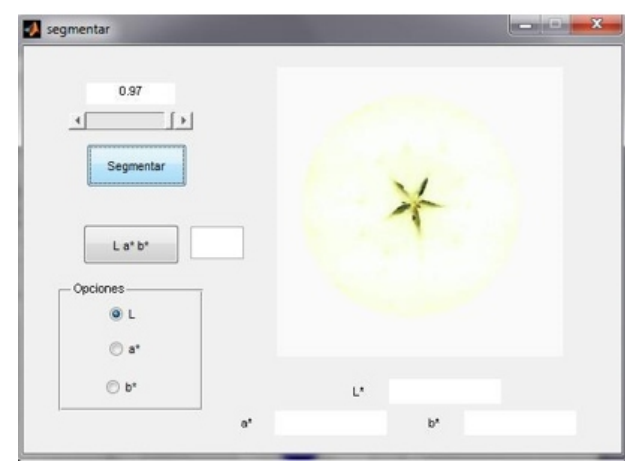

b)

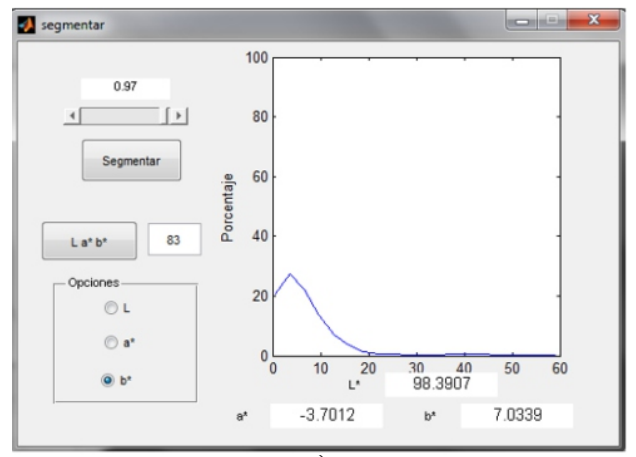

c)

Figura 4. Extracción de información 


\section{Modelado de los cambios en el color, la temperatura y el pre-tratamiento.}

El análisis de la relación entre los parámetros de color, temperatura y pre-tratamiento requirió de los siguientes pasos:

- Análisis de la normalidad, determinando mediante la validez estadística de la data recolectada.

- Análisis de varianza, determinando el efecto estadístico de cada uno de los factores (temperatura, pre-tratamiento y tiempo) así como sus combinaciones en los valores medios de los parámetros de color.

- Modelado, se aplicó el análisis estadístico mediante regresiones lineales múltiples, determinando de esta manera modelos lineales aditivos de acuerdo con la ecuación 1 :

Donde

$$
\Psi=a_{1} \cdot P_{1}+a_{2} . P_{2}+a_{3} \cdot P_{3}+\varepsilon \ldots . \text { Ecuación } 1
$$

$\Psi=$ Variable dependiente (color en coordenadas L, $\mathrm{a}^{*}, \mathrm{~b}^{*}$ ) $\mathrm{a}_{\mathrm{i}}=$ Constante de la variable independiente ${ }_{\mathrm{i}}$

$\mathrm{P}_{\mathrm{i}}=$ Variable independiente $\mathrm{i}$; $\left(\mathrm{T}^{\mathrm{o}}\right.$, Pre-tratamiento, tiempo) $\varepsilon=$ Error

\section{RESULTADOS}

4.1. Imágenes del proceso de pardeamiento. Un ejemplo de las imágenes obtenidas se muestra en la Figura 5 en la cual cada columna significa un tratamiento y las filas corresponden al tiempo.

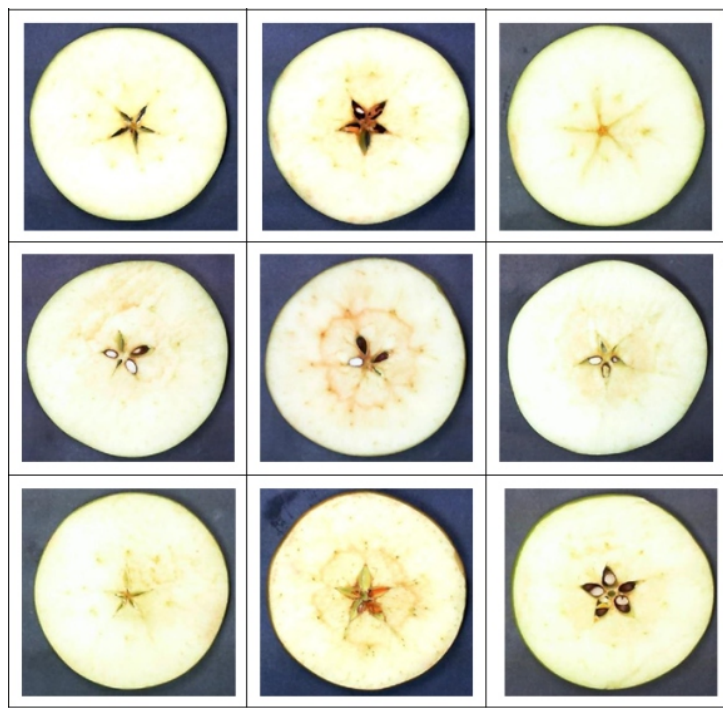

Figura 5. Imágenes de rodajas de manzanas durante tratamiento

De las imágenes obtenidas se aprecia que, al iniciar, durante y al finalizar el tiempo de estudio, existen diferencias entre los tratamientos y el color de las muestras debido al proceso de pardeamiento enzimático.
4.2.Variación de los parámetros de color durante el proceso de pardeamiento. En las siguientes figuras se muestran las gráficas de los valores medios de los parámetros $\mathrm{L}, \mathrm{a}^{*} \mathrm{y} \mathrm{b}^{*}$.

Parámetro L. Como se observa en la Figura 6a para el parámetro L existe una ligera tendencia decreciente en las muestras sin pre-tratamiento durante el proceso de pardeamiento enzimático; se aprecia, asimismo, que las muestras sometidas a una temperatura de 25 ${ }^{\circ} \mathrm{C}$ tienen la mayor diminución de este parámetro de color. En la Figura 6b, al igual que en la Figura 6a, se aprecia que el parámetro L tiene tendencia decreciente; sin embargo, es más pronunciada que en las muestras sin tratamiento y no se aprecia que existan diferencias sensibles entre las temperaturas. Por último, en la Figura $6 \mathrm{c}$ se aprecia que en las muestras sulfitadas casi no ha existido cambio en el parámetro L.

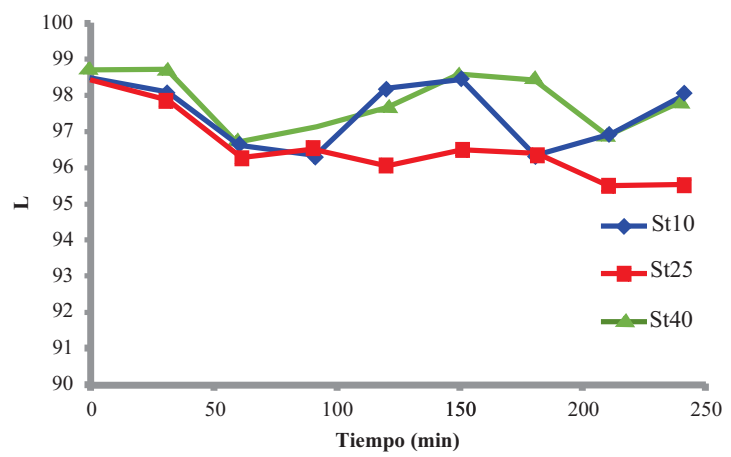

a)

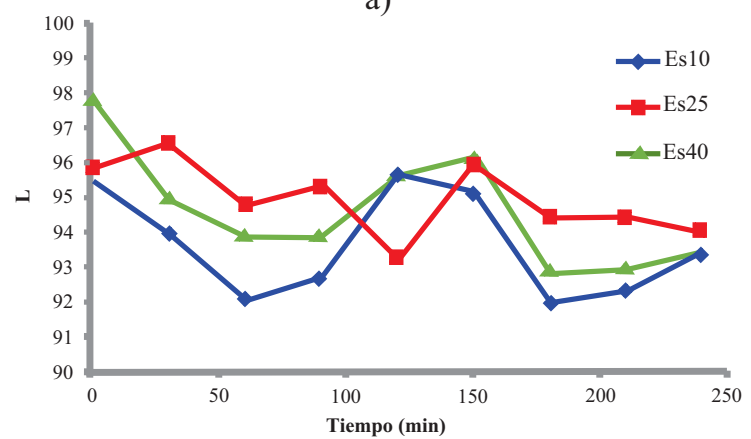

b)

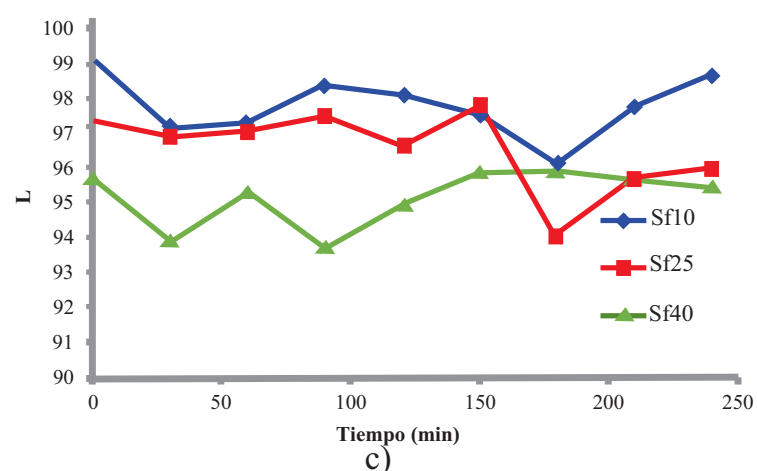

Figura 6: Valor promedio de L en muestras a) sin tratamiento b) escaldadas y c) sulfitadas 
Por tanto; el parámetro L para todas las muestras decrece, es decir estas pierden luminosidad, a lo largo del periodo de evaluación, siendo más pronunciado en aquellas muestras que se almacenaron a $25^{\circ} \mathrm{C}$. Salvo en el caso de las muestras escaldadas en las cuales la temperatura de $40{ }^{\circ} \mathrm{C}$ incrementa velocidad de reducción de la luminosidad.

Parámetro $a *$ En la Figura 7a se aprecia que existieron cambios en el valor del parámetro a*, tendencia creciente, de esto se infiere que existió la disminución de las tonalidades verdes e incremento de tonalidades rojizas. En la Figura $7 b$ se aprecia que los valores de a* para las muestras escaldadas se mantuvo prácticamente constante, verificándose posteriormente este resultado con el análisis estadístico.

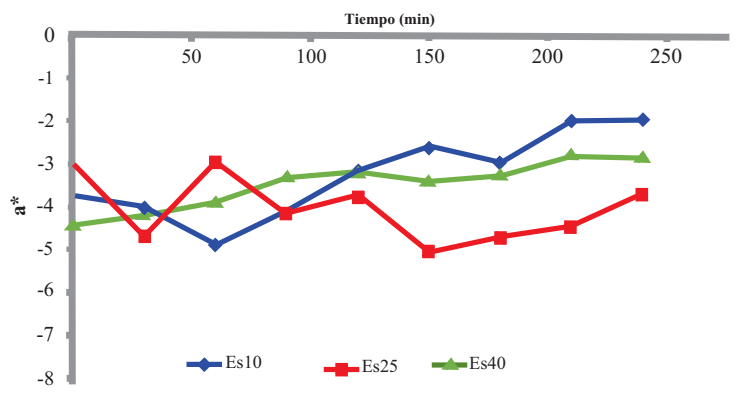

a)

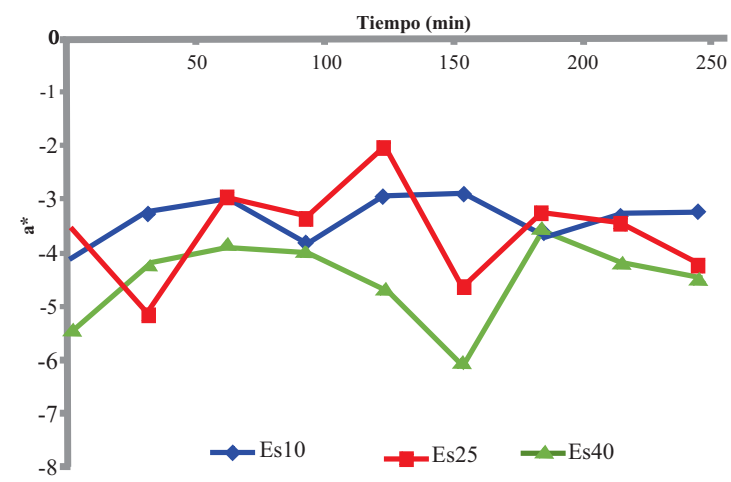

b)

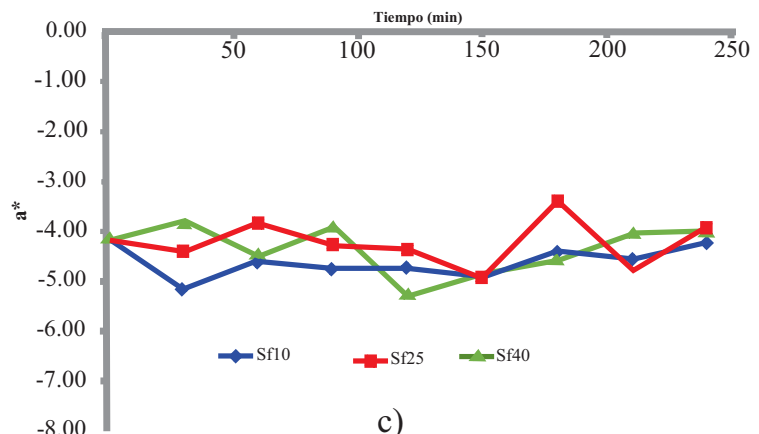

Figura 7. Valor promedio de $a^{*}$ en muestras a) $\sin$ tratamiento b) escaldadas y c) sulfitadas
De todo lo anterior se aprecia que el parámetro a* aumenta, reducción de tonalidades verdes e incremento de tonalidades rojas; sin embargo, es un incremento poco sensible en todos los casos.

Parámetro $\boldsymbol{b}$ *. En las Figuras 8 a a $8 \mathrm{c}$ se aprecia que existieron cambios en el valor del parámetro b*, El parámetro $b^{*}$ se incrementa, aumento de tonalidades amarillas, a lo largo del proceso de evaluación, siendo más pronunciado en temperaturas de $40^{\circ} \mathrm{C}$.

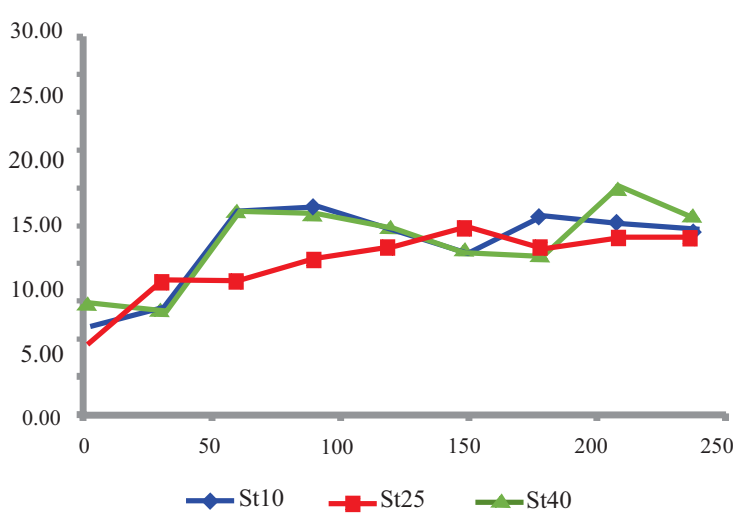

a)

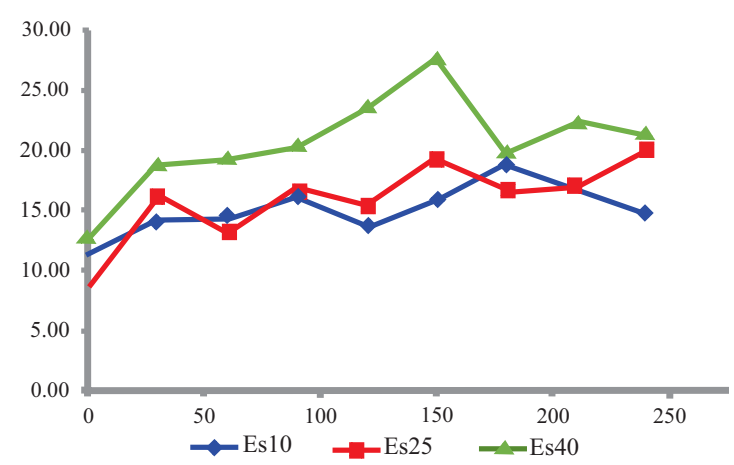

b)

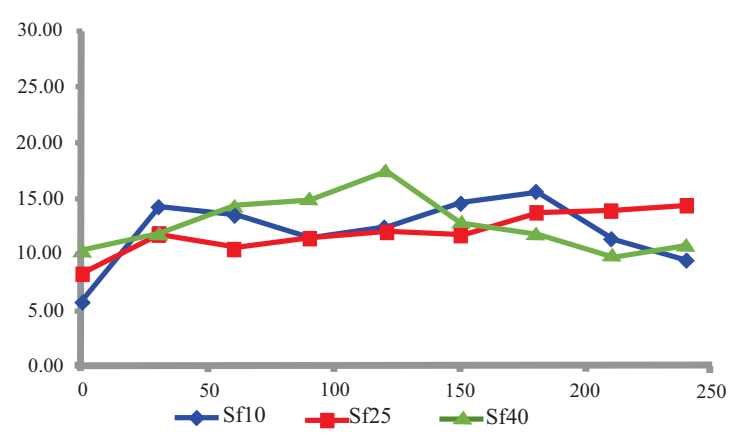

c)

Figura 8. Valor promedio de $b^{*}$ en muestras a) $\sin$ tratamiento b) escaldadas y c) sulfitadas 


\subsection{Modelado}

Parámetro $L$. La salida muestra los resultados del ajuste a un modelo de regresión lineal múltiple para describir la relación entre L y 3 variables independientes, Figura 9a. La ecuación del modelo ajustado es:

$\mathrm{L}=97.4672-0.411481 * \mathrm{P}-0.014 * \mathrm{Ta} 0.00521235 * \mathrm{Tp}$ Donde:

$$
\begin{aligned}
& \mathrm{P}=\text { pre-tratamiento } \\
& \mathrm{Tp}=\text { tiempo } \\
& \mathrm{Ta}=\text { temperatura de almacenamiento }
\end{aligned}
$$

El R-cuadrado indica que el modelo explica un $9.7278 \%$ de la variabilidad en L. Dado que el p-valor es inferior a 0.05 , hay indicio de una posible correlación serial. Para simplificar el modelo se usa el p-valor, puesto que el p-valor para la temperatura es superior o igual a 0.10 , este término no es estadísticamente significativo para un nivel de confianza del $90 \%$ o superior; por tanto, se puede retirar Temperatura del modelo.

Parámetro $a *$ La salida muestra los resultados del ajuste a un modelo de regresión lineal múltiple para describir la relación entre $a^{*}$ y 3 variables independientes, Figura 9b. La ecuación del modelo ajustado es:

$\mathrm{a}^{*}=-3.46857-0.398 * \mathrm{P}-0.012037 * \mathrm{Ta}+0.00184815^{*} \mathrm{Tp}$ Donde:

$$
\begin{aligned}
& \mathrm{P}=\text { pre-tratamiento } \\
& \mathrm{Tp}=\text { tiempo } \\
& \mathrm{Ta}=\text { temperatura de almacenamiento }
\end{aligned}
$$

El R-cuadrado indica que el modelo explica un $23.2105 \%$ de la variabilidad en $\mathrm{a}^{*}$. Puesto que los $\mathrm{p}$ valores son inferiores a 0.01 no se recomienda simplificar el modelo.

Parámetro $\boldsymbol{b}^{*}$. La salida muestra los resultados del ajuste a un modelo de regresión lineal múltiple para describir la relación entre $b^{*}$ y 3 variables independientes, Figura 9c. La ecuación del modelo ajustado es:

$\mathrm{b}^{*}=10.3903-0.4067 * \mathrm{P}+0.07186 * \mathrm{Ta}+0.0198426^{*} \mathrm{Tp}$ Donde:

$$
\begin{aligned}
& \mathrm{P}=\text { pre-tratamiento } \\
& \mathrm{Tp}=\text { tiempo } \\
& \mathrm{Ta}=\text { temperatura de almacenamiento }
\end{aligned}
$$

El estadístico R-cuadrado indica que el modelo explica un $21.748 \%$ de la variabilidad en b. Para la simplificación del modelo, puesto que el p-valor de pre- tratamiento, es superior o igual a 0.10 , este término no es estadísticamente significativo para un nivel de confianza del $90 \%$ o superior; por tanto, es posible retirar pre-tratamiento del modelo.

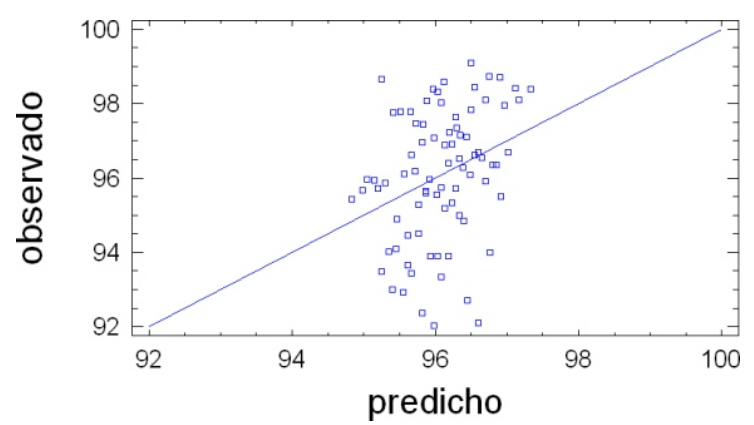

a)

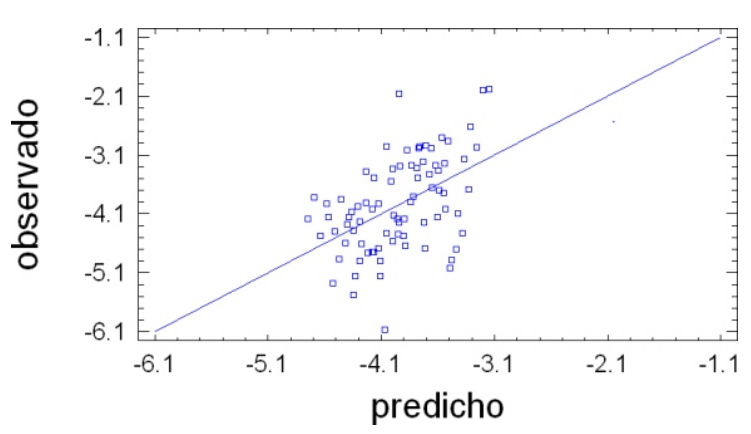

b)

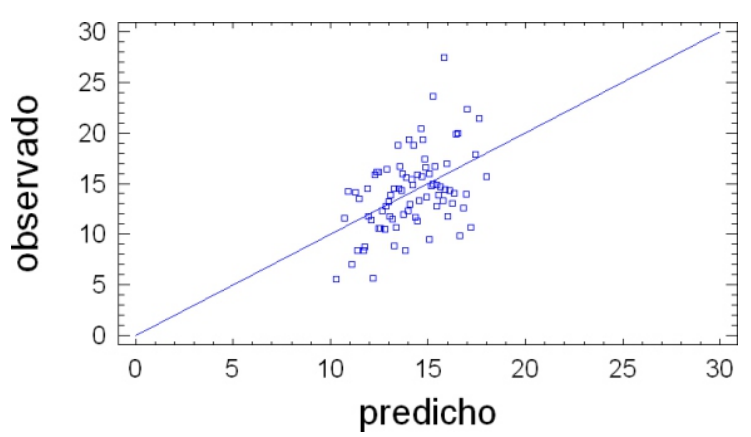

c)

Figura 9. Parámetro predicho vs observado a) L, b) $\left.a^{*}, b\right) b^{*}$

\section{DISCUSIÓN}

De las Figura 5 se aprecia que las muestras presentaron el típico color pardo producto de la reacción enzimática de pardeamiento cuyo como catalizador es la polifenol oxidasa (PFO), la cual combina los fenoles con el oxígeno para transformarse en quinonas, que se polimerizan o reaccionan con grupos amino de diferentes compuestos formando compuestos coloridos que reciben el nombre de melaninas y que tienen propiedades antimicrobianas, y que podrían ser un mecanismo de defensa de los vegetales contra infecciones (Luo, 1997). 
En la Figura 6, se observa la variación de los valores promedio de $\mathrm{L}$ en función del tiempo donde se aprecia la disminución de dichos valores debido al oscurecimiento de las mismas. El oscurecimiento de frutas y verduras crudas es debido principalmente a lesiones mecánicas durante el manejo postcosecha y procesamiento. Esta reacción resulta de la acción de la polifenol-oxidasa (PPO), oxidación catalizada por compuestos fenólicos a o quinonas, que posteriormente se polimerizan para formar pigmentos de color oscuro (Sapers, 1987). De hecho, cuando las frutas se cortan, la superficie de corte se vuelve marrón, reduce no sólo la calidad visual, sino también da lugar a cambios indeseables en el sabor y la pérdida de nutrientes debido a la oxidación enzimática (Luo, 1997).

La disminución del valor de L se debe principalmente a la falta de uniformidad patrones de color que surgen debido al pardeamiento enzimático que causa diferencias significativas entre los valores de luminosidad de las imágenes, este fenómeno provoca que el valor de L sea cada vez más irregular o complejo, este comportamiento es debido al hecho de que algunas zonas de las rodajas de manzana se oscurecen más rápido que otros, causados por una reacción enzimática no homogénea en toda la muestra. De acuerdo con lo reportado, en la literatura, por otros investigadores los valores L, disminuyen durante el pardeamiento enzimático en rodajas de fruta. (Lu et al, 2007; Luo, 1994, 1997; Sapers, 1987).

También apreciamos en la Figura 7, que la mayoría de los valores de las coordenadas a* con el transcurso del tiempo se incrementan esto indica que las imágenes de rodajas de manzana obtenidas mediante visión computacional tienden al color rojo. Estos resultados coinciden con los encontrados por otros autores para el análisis del cambio de color de rodajas de patata de variedades como Panda, Saturna y Bintje (Pedreschi et al, 2004; Segnini et al., 1999).

La variación del parámetro $b^{*}$ fue más marcada que para el parámetro $a^{*}$, es decir se aprecia la que a medida que aumenta el tiempo, las rodajas de manzana tienden al color amarillo. Estos resultados sugieren que la amarillez de rodajas de manzana aumenta durante el tiempo y coinciden con los obtenidos por otros autores (Krokida et al., 2001; Segniniet al., 1999).

\section{CONCLUSIONES}

De acuerdo con los resultados obtenidos es posible hacer el seguimiento de los cambios en los parámetros de color, expresados en el espacio
CIELa*b*, mediante un sistema de visión artificial. Asimismo, se observa que, a lo largo del proceso de pardeamiento se produce la reducción de los parámetros Le incremento de parámetros $\mathrm{a}^{*} \mathrm{y} \mathrm{b}^{*}$.

Los modelos confirman que los parámetros L, a* y b* tienen relación con los factores en evaluación. Sin embargo, por el bajo valor de R2, no es posible utilizarlos para predecir el proceso.

\section{REFERENCIAS BIBLIOGRÁFICAS}

Briones \& Aguilera (2005). Image analysis of changes in surface color of chocolate. Food Research International.Brosnan \& Sun. (2004). Improving quality inspection of food products by computer vision. Journal of Food Engineering, 125-135.

Du \& Sun. (2005). Pizza sauce spread classification using colour vision and support vector machines. Journal of Food Engineering.

Guoping, y Col. (2004). Compressing histogram representations for automatic colour photo categorization. Pattern Recognition.

Kang \& Sabarez. (2009). Simple colour image segmentation of bicolour food products for quality mesurement. Journal of Food Engineering.

Kit \& Spyridon. (2004). A simple digital imaging method for measuring and analyzing color of food surfaces. Journal of Food Engineering.

Krokida, et al.; (2001). Colour changes during deep far frying. Journal of Food Engineering. 219-225.

León. (2005). Estudio de la cinética de color en rodajas de papa frita empleando visión computacional. Facultad Tecnológica. Universidad de Santiago de Chile: Trabajo de titulación para optar al título de Ingeniero de Alimentos. Departamento de Ciencia y Tecnología de los Alimentos.

Lu et al.; (2007). Efficacy of sodium chlorite as an inhibitor of enzymatic browning in apple slices. Food Chemistry. 824-829.

Lunadei, \& Col. (2011). A multispectral vision system to evaluate enzymatic browning in fresh-cut apple slices. Postharvest Biology and Technology.

Luo. 1994. Enhanced control of enzymatic browning of apple slices by papain.

Luo. 1997. Enzymatic browning and its inhibition in new apple cultivars slices using 4hexylresorcinol in combination with ascorbic acid. Food Science and Technology International. 195-201.

Nicolas et al.; (1994). Enzymatic browning reactions in apple and apple products. CRC Crit.Rev. 
Food. Sci.. 109-157.

Paschos \& Petrou. (2003). Histogram ratio features for color texture classification. Pattern Recognition Letters.

Pedreschi et al.; (2004). Classification of potato chips using pattern recognition. Journal of Food Science. 264-270.

Robards et al.; (1999). Phenolic compounds and their role in oxidative processes in fruits. Food Chemistry. 66. 401-436.

Sapers, 1987. Comparison of erythorbic and ascorbic acids as inhibitors of enzymatic browning in apple. Journal of Food Science. 17-32.

Segnini et al., (1999). A low cost video technique for color measurement of potato chips. Lebensmittel-Wissenschaft und Technologie. 216-222.

Xul \& Col.; (2009). On-Line detection of defects on fruit by machinevision systems based on three-color-cameras systems. Computer and Computing Technologies in Agriculture 2231-2238

Yoo \& Il-Seok (1999). A fast algorithm for tracking human faces based on chromatic histograms. Pattern Recognition Letters 